\title{
On the average of character sums for a group of characters
}

\author{
by
}

\author{
D. A. Burgess (Nottingham)
}

To J. W. S. Cassels

1. Introduction. Let $r, k, h$ be positive integers. For any Dirichlet character $\chi$ modulo $k$ write

$$
W_{r}(\chi)=\sum_{x=1}^{k}\left|\sum_{m=1}^{h} \chi(x+m)\right|^{2 r} .
$$

In [2] it was shown that, if $\chi$ is a primitive character, then

$$
W_{2}(\chi) \ll k h^{2}+k^{1 / 2+\varepsilon} h^{4},
$$

which implies that

$$
W_{2}(\chi) \ll k^{1+\varepsilon} h^{2}
$$

for $h \leq k^{1 / 4}$. In [6] it was shown that

$$
\sum_{\text {primitive } \chi(\bmod k)} W_{2}(\chi) \ll k^{2+\varepsilon} h^{2},
$$

so that (2) holds for all $h$, on average for all primitive characters modulo $k$. Thus it is reasonable to conjecture that (2) might hold for some $h>k^{1 / 4}$, on average for the primitive characters of a large subgroup of the characters modulo $k$. In [8] such a result was obtained, it being shown that, for any prime $p$,

$$
\sum_{\substack{\chi \bmod p^{3} \\ \chi^{p^{2}}=\chi_{0}}} W_{2}(\chi) \ll p^{5} h^{2}+p^{3} h^{4},
$$

where $\chi_{0}$ is the principal character, and thus (2) holds for $h \leq k^{1 / 3}$, on average for the characters modulo $k=p^{3}$ in the group of order $p^{2}$.

1991 Mathematics Subject Classification: Primary 11L40. 
In this paper the argument is strengthened to show in the following theorem that, for the non-principal characters of this group, (2) remains true for $h \leq k^{1 / 2}$, on average.

TheOREM 1. Let $p$ be an odd prime number. Let

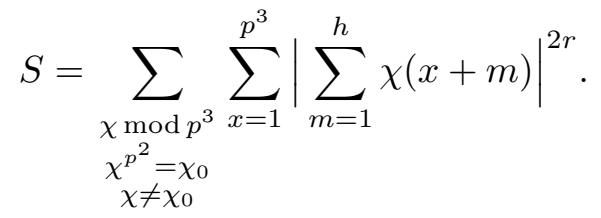

Then in the case $r=2$ we have

$$
S \ll p^{2} h^{4}+p^{5} h^{2} .
$$

From [1] it follows that if $k$ is prime then

$$
W_{3}(\chi) \ll k h^{3}+k^{1 / 2} h^{6}
$$

for all positive $h$. By the methods of this paper it is shown that (5) can be improved for $h>p^{3 / 4}$, on average for the non-principal characters modulo $k=p^{3}$ in the group of order $p^{2}$.

TheOREM 2. Let $p$ be an odd prime number. Let

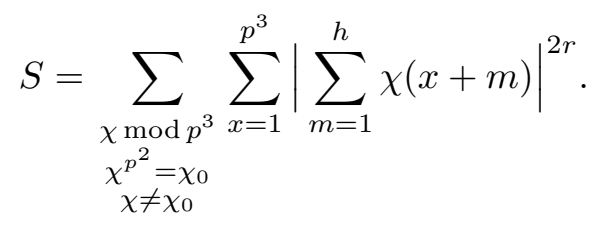

Then in the case $r=3$ we have

$$
S \ll p^{2} h^{6}+\min (h, p)^{3} p^{5} h+\min (h, p)^{2} p^{6} .
$$

In Section 8 we shall describe some corollaries of these theorems.

2. Preliminary transformation of the problem. For $S$ as in the statement of both theorems, we have

(6) $S=\sum_{\substack{\chi \bmod p^{3} \\ \chi^{p^{2}}=\chi_{0}}} \sum_{x=1}^{p^{3}}\left|\sum_{m=1}^{h} \chi(x+m)\right|^{2 r}-\sum_{x=1}^{p^{3}}\left(\sum_{m=1}^{h} \chi_{0}(x+m)\right)^{2 r}=S_{1}-S_{2}$, say.

Now

$$
S_{2}=\sum_{\boldsymbol{m}} \sum_{x=1}^{p^{3}} \chi_{0}\left(f_{1}(x)\right) \chi_{0}\left(f_{2}(x)\right)
$$

where $\boldsymbol{m} \in \mathbb{Z}^{2 r}$ satisfies $0<m_{i} \leq h$ for $1 \leq i \leq 2 r$, and 


$$
f_{1}(x)=\left(x+m_{1}\right) \ldots\left(x+m_{r}\right), \quad f_{2}(x)=\left(x+m_{r+1}\right) \ldots\left(x+m_{2 r}\right) .
$$

Thus

(7) $S_{2}=\sum_{\boldsymbol{m}} \sum_{\substack{x=1 \\ x \neq \equiv-m_{i}(\bmod p)}}^{p^{3}} 1=\sum_{\boldsymbol{m}} p^{2} \#\left\{x: 1 \leq x \leq p, p \nmid f_{1}(x) f_{2}(x)\right\}$.

We also have

$$
\begin{aligned}
S_{1}= & \sum_{\boldsymbol{m}} \sum_{x=1}^{p^{3}} \sum_{\chi^{p^{2}=\chi_{0}}} \chi\left(f_{1}(x)\right) \bar{\chi}\left(f_{2}(x)\right)=\sum_{\boldsymbol{m}} \sum_{\substack{x=1 \\
p \nmid f_{1}(x) f_{2}(x)}}^{p^{3}} \sum_{p^{p^{2}=\chi_{0}}} \chi\left(\frac{f_{1}(x)}{f_{2}(x)}\right) \\
= & p^{2} \sum_{\boldsymbol{m}} \#\left\{1 \leq x \leq p^{3}, 1 \leq z<p: p \nmid f_{1}(x) f_{2}(x),\right. \\
& \left.f_{1}(x)-z^{p^{2}} f_{2}(x) \equiv 0\left(\bmod p^{3}\right)\right\} .
\end{aligned}
$$

Thus, writing

$$
f_{(z)}(x)=f_{1}(x)-z^{p^{2}} f_{2}(x)
$$

we have

$$
S_{1}=S_{3}+S_{4}
$$

where

(10) $S_{3}=p^{2} \sum_{\boldsymbol{m}} \sum_{z=1}^{p-1} \#\left\{1 \leq x \leq p^{3}: x \not \equiv-m_{i}(\bmod p)\right.$,

$$
\left.f_{(z)}(x) \equiv 0\left(\bmod p^{3}\right), f_{(z)}^{\prime}(x) \not \equiv 0(\bmod p)\right\}
$$

and

$$
\begin{aligned}
S_{4}=p^{2} \sum_{m} \sum_{z=1}^{p-1} \#\left\{1 \leq x \leq p^{3}: x \neq-m_{i}(\bmod p),\right. \\
\left.f_{(z)}(x) \equiv 0\left(\bmod p^{3}\right), f_{(z)}^{\prime}(x) \equiv 0(\bmod p)\right\} .
\end{aligned}
$$

We consider the non-singular roots of $f_{(z)}$. Since the numbers of nonsingular roots modulo $p^{3}$ and $p$ are the same we have from (10) that

$$
\begin{aligned}
S_{3} & \leq p^{2} \sum_{\boldsymbol{m}} \sum_{z=1}^{p-1} \#\left\{1 \leq x \leq p: p \nmid f_{1}(x) f_{2}(x), f_{(z)}(x) \equiv 0(\bmod p)\right\} \\
& =p^{2} \sum_{\boldsymbol{m}} \sum_{\substack{x=1 \\
p \nmid f_{1}(x) f_{2}(x)}}^{p} \#\left\{1 \leq z<p: f_{1}(x)-z f_{2}(x) \equiv 0(\bmod p)\right\} \\
& =p^{2} \sum_{\boldsymbol{m}} \#\left\{1 \leq x \leq p: p \nmid f_{1}(x) f_{2}(x)\right\}=S_{2}
\end{aligned}
$$


from (7). Now from (6) and (9) we have

$$
S \leq S_{4} .
$$

3. Estimates for solution sets of polynomials. In the proof of our theorems we shall require some lemmas concerning the number of solutions of congruences to a prime power modulus. We shall use the following generalisation of the well known estimate for the number of non-singular roots of a congruence.

LEMMA 1. Let $F$ be a polynomial of degree $n$ having integer coefficients. Let $p$ be a prime, $d$ a positive integer, and $\alpha, \beta$ and $\gamma$ be non-negative integers satisfying $\gamma=\lceil\alpha / d\rceil$. Then

$$
\#\left\{1 \leq x \leq p^{\gamma}: p^{\alpha+\beta} \mid F(x), p^{\beta} \| F^{(d)}(x)\right\} \leq n .
$$

Proof. This is Proposition 1 of [7].

We shall require an estimate for the number of solutions of a congruence in many variables to a prime modulus. The following will suffice.

LEMma 2. Let $G$ be a polynomial in $x_{1}, \ldots, x_{t}$ which is not identically zero modulo the prime $p$. Let $0<h_{i} \leq p$ for all $i$. Then the number of $\boldsymbol{x}$, satisfying $0<x_{i} \leq h_{i}$ for all $i$, for which $G(\boldsymbol{x}) \equiv 0(\bmod p)$ is $O\left(\left(\prod h_{i}\right) / \min h_{i}\right)$.

Proof. This is an easy modification of Lemma 5 of [4].

We shall also use the following estimate which, under favourable conditions, can provide an optimal estimate for the average number of singular roots of a set of polynomials.

Lemma 3. Let $F_{i}(\boldsymbol{y})(0<i \leq n)$ be polynomials in $\nu$ variables $y_{k}(0<$ $k \leq \nu)$. Let $p$ be a prime and $2 \beta \geq \alpha_{1} \geq \ldots \geq \alpha_{m}>\beta \geq \alpha_{m+1} \geq \ldots \geq \alpha_{n}$ be positive integers. Let $\boldsymbol{H} \in \mathbb{N}^{\nu}$. Write

$$
N=\#\left\{\boldsymbol{y}: \forall k \leq \nu 0<y_{k} \leq H_{k}, \forall i F_{i}(\boldsymbol{y}) \equiv 0\left(\bmod p^{\alpha_{i}}\right)\right\} .
$$

Put $\lambda_{k}=\left\lceil H_{k} / p^{\beta}\right\rceil$ for all $k \leq \nu$. Then

$$
\begin{gathered}
N \ll \sum_{\substack{B \\
\forall k \leq \nu\left|B_{k}\right|<\lambda_{k}}} \#\left\{\boldsymbol{y}: \forall k \leq \nu 0<y_{k} \leq p^{\beta}, \forall i \leq m F_{i}(\boldsymbol{y}) \equiv 0\left(\bmod p^{\beta}\right),\right. \\
\left.\forall i>m F_{i}(\boldsymbol{y}) \equiv 0\left(\bmod p^{\alpha_{i}}\right), \forall i \leq m \sum_{k=1}^{\nu} B_{k} \frac{\partial F_{i}}{\partial y_{k}}(\boldsymbol{y}) \equiv 0\left(\bmod p^{\alpha_{i}-\beta}\right)\right\} .
\end{gathered}
$$

Pr o of. Clearly

$$
N \leq \#\left\{\boldsymbol{y}: \forall k \leq \nu 0<y_{k} \leq \lambda_{k} p^{\beta}, \forall i F_{i}(\boldsymbol{y}) \equiv 0\left(\bmod p^{\alpha_{i}}\right)\right\} .
$$


For $1 \leq k \leq \nu$ write $y_{k}=a_{k}+p^{\beta} b_{k}$, where $0<a_{k} \leq p^{\beta}, 0 \leq b_{k}<\lambda_{k}$. Then, for $m<i \leq n, F_{i}(\boldsymbol{y}) \equiv 0\left(\bmod p^{\alpha_{i}}\right)$ becomes

$$
F_{i}(\boldsymbol{a}) \equiv 0\left(\bmod p^{\alpha_{i}}\right),
$$

while for $i \leq m, F_{i}(\boldsymbol{y}) \equiv 0\left(\bmod p^{\alpha_{i}}\right)$ becomes

$$
F_{i}(\boldsymbol{a})+\sum_{k=1}^{\nu} \frac{\partial F_{i}}{\partial y_{k}}(\boldsymbol{a}) b_{k} p^{\beta} \equiv 0\left(\bmod p^{\alpha_{i}}\right) .
$$

The latter congruences imply, for $i \leq m$,

$$
F_{i}(\boldsymbol{a}) \equiv 0\left(\bmod p^{\beta}\right)
$$

so that, say,

$$
\forall i \leq m \quad F_{i}(\boldsymbol{a})=c_{i}(\boldsymbol{a}) p^{\beta}
$$

Thus we have

$$
\begin{aligned}
N \leq \sum_{\substack{\boldsymbol{a} \\
\forall i \leq m F_{i}(\boldsymbol{a}) \equiv 0\left(\bmod p^{\beta}\right) \\
\forall i>m F_{i}(\boldsymbol{a}) \equiv 0\left(\bmod p^{\alpha_{i}}\right)}} \#\left\{\boldsymbol{b}: \forall k \leq \nu 0 \leq b_{k}<\lambda_{k},\right. \\
\left.\forall i \leq m \sum_{k=1}^{\nu} \frac{\partial F_{i}}{\partial y_{k}}(\boldsymbol{a}) b_{k} \equiv-c_{k}(\boldsymbol{a})\left(\bmod p^{\alpha_{i}-\beta}\right)\right\} .
\end{aligned}
$$

Now the number of solutions of the inhomogeneous congruences

$$
\forall i \leq m \quad \sum_{k=1}^{\nu} \frac{\partial F_{i}}{\partial y_{k}}(\boldsymbol{a}) b_{k} \equiv-c_{k}(\boldsymbol{a})\left(\bmod p^{\alpha_{i}-\beta}\right)
$$

in the variables $\boldsymbol{b}$ satisfying $0 \leq b_{k}<\lambda_{k}$ for all $k \leq \nu$ is at most the number of solutions of the homogeneous congruences

$$
\forall i \leq m \quad \sum_{k=1}^{\nu} \frac{\partial F_{i}}{\partial y_{k}}(\boldsymbol{a}) B_{k} \equiv 0\left(\bmod p^{\alpha_{i}-\beta}\right)
$$

in the variables $\boldsymbol{B}$ satisfying $\left|B_{k}\right|<\lambda_{k}$ for all $k \leq \nu$. Thus we have

$$
\begin{gathered}
N \leq \sum_{\substack{\forall i \leq m F_{i}(\boldsymbol{a}) \equiv 0\left(\bmod p^{\beta}\right) \\
\forall i>m F_{i}(\boldsymbol{a}) \equiv 0\left(\bmod p^{\alpha_{i}}\right)}} \#\left\{\boldsymbol{B}: \forall k \leq \nu\left|B_{k}\right|<\lambda_{k},\right. \\
\leq \sum_{\substack{\boldsymbol{B} \\
\forall k \leq \nu\left|B_{k}\right|<\lambda_{k}}} \#\left\{\boldsymbol{a}: \forall k \leq \nu 0<a_{k=1}^{\nu} \frac{\partial F_{i}}{\partial y_{k}}(\boldsymbol{a}) B_{k} \equiv 0\left(\bmod p^{\alpha_{i}-\beta}\right)\right\} \\
\forall i>m F_{i}(\boldsymbol{a}) \equiv 0\left(\bmod p^{\alpha_{i}}\right), \forall i \leq m F_{i}(\boldsymbol{a}) \equiv 0\left(\bmod p^{\beta}\right), \\
\left.\forall i \sum_{k=1}^{\nu} B_{k} \frac{\partial F_{i}}{\partial y_{k}}(\boldsymbol{a}) \equiv 0\left(\bmod p^{\alpha_{i}-\beta}\right)\right\} .
\end{gathered}
$$


4. Proof of Theorem 1. Clearly in proving the theorem we may suppose that $p>2$. We consider here the case $r=2$.

It remains to consider the singular roots. Noting (11) we write

$$
S_{4}=S_{5}+S_{6},
$$

where

$$
\begin{aligned}
S_{5}=p^{2} \sum_{\boldsymbol{m}} \#\left\{1 \leq x \leq p^{3}, 1 \leq z<p: x \not-m_{i}(\bmod p),\right. \\
\left.f_{(z)}(x) \equiv 0\left(\bmod p^{3}\right), f_{(z)}^{\prime}(x) \equiv 0(\bmod p), f_{(z)}^{\prime \prime}(x) \equiv 0(\bmod p)\right\},
\end{aligned}
$$

and

$$
\begin{array}{r}
S_{6}=p^{2} \sum_{m} \sum_{z=2}^{p-1} \#\left\{1 \leq x \leq p^{3}: x \neq-m_{i}(\bmod p), f_{(z)}(x) \equiv 0\left(\bmod p^{3}\right),\right. \\
\left.f_{(z)}^{\prime}(x) \equiv 0(\bmod p), f_{(z)}^{\prime \prime}(x) \not \equiv 0(\bmod p)\right\} .
\end{array}
$$

Clearly we have

$$
\begin{aligned}
S_{5} \leq p^{2} \sum_{\boldsymbol{m}} \#\left\{1 \leq x \leq p^{3}:\right. & \\
\left(m_{1}+m_{2}-m_{3}-m_{4}\right) x+\left(m_{1} m_{2}-m_{3} m_{4}\right) & \equiv 0\left(\bmod p^{3}\right), \\
\left(m_{1}+m_{2}-m_{3}-m_{4}\right) & \equiv 0(\bmod p)\} .
\end{aligned}
$$

Write

$$
p^{\delta}=\text { highest common factor }\left(p^{3}, m_{1}+m_{2}-m_{3}-m_{4}\right),
$$

where $1 \leq \delta \leq 3$. For solubility of the congruence

$$
\left(m_{1}+m_{2}-m_{3}-m_{4}\right) x+\left(m_{1} m_{2}-m_{3} m_{4}\right) \equiv 0\left(\bmod p^{3}\right)
$$

we require also

$$
p^{\delta} \mid\left(m_{1} m_{2}-m_{3} m_{4}\right) .
$$

The congruence then has at most $p^{\delta}$ solutions satisfying $1 \leq x \leq p^{3}$. (14) and (15) imply

$$
\left(m_{1}-m_{3}\right)\left(m_{2}-m_{3}\right) \equiv 0\left(\bmod p^{\delta}\right) .
$$

Suppose that $p^{\varepsilon} \mid\left(m_{1}-m_{3}\right)$ and $p^{\delta-\varepsilon} \mid\left(m_{2}-m_{3}\right)$. Then the number of such $\boldsymbol{m}$ is

$$
O\left(h\left(1+\frac{h}{p^{\varepsilon}}\right)\left(1+\frac{h}{p^{\delta-\varepsilon}}\right)\left(1+\frac{h}{p^{\delta}}\right)\right)=O\left(\frac{h^{4}}{p^{2 \delta}}+h^{2}\right) .
$$

Thus

$$
S_{5} \ll p^{2} \sum_{\delta, \varepsilon} p^{\delta}\left(\frac{h^{4}}{p^{2 \delta}}+h^{2}\right) \ll p h^{4}+p^{5} h^{2} .
$$


On the other hand, we have

$$
\begin{aligned}
S_{6} \ll p^{2} \sum_{\boldsymbol{m}} \sum_{z=2}^{p-1} \#\left\{1 \leq x \leq p^{3}: f_{(z)}(x)\right. & \equiv 0\left(\bmod p^{3}\right), \\
f_{(z)}^{\prime}(x) & \left.\equiv 0(\bmod p), f_{(z)}^{\prime \prime}(x) \not \equiv 0(\bmod p)\right\} .
\end{aligned}
$$

Thus, by Lemma 1 ,

$$
S_{6} \ll p^{3} \#\left\{\boldsymbol{m}, 1<z<p: \exists x f_{(z)}(x) \equiv 0\left(\bmod p^{2}\right), f_{(z)}^{\prime}(x) \equiv 0(\bmod p)\right\} .
$$

Thus

$$
\begin{aligned}
S_{6} \ll p^{3} \#\left\{\boldsymbol{m}, z, x: \forall i 0<m_{i} \leq h, 0<x \leq p, 0<z<p,\right. \\
\left.f_{(z)}(x) \equiv 0\left(\bmod p^{2}\right), f_{(z)}^{\prime}(x) \equiv 0(\bmod p)\right\} .
\end{aligned}
$$

Put

$$
\lambda=\left\lceil\frac{h}{p}\right\rceil, \quad \mu= \begin{cases}p & \text { if } \lambda>1 \\ h & \text { if } \lambda=1\end{cases}
$$

Now we apply Lemma 3 , treating $x, z$ as constants and the $m_{i}$ as our variables, to obtain

$$
\begin{array}{r}
S_{6} \ll p^{3} \sum_{\substack{B \\
\forall k \leq 4\left|B_{k}\right|<\lambda}} \#\left\{\boldsymbol{a}, x, z: \forall k \leq 40<a_{k} \leq \mu, 0<x \leq p,\right. \\
0<z<p, f_{(z)}(x) \equiv 0(\bmod p), \\
\left.f_{(z)}^{\prime}(x) \equiv 0(\bmod p), \sum_{k=1}^{4} B_{k} \frac{\partial f_{(z)}}{\partial m_{k}}(x) \equiv 0(\bmod p)\right\}
\end{array}
$$

if $\lambda>1$, while if $\lambda=1$ this follows immediately from (17).

Given $\boldsymbol{B}, x, z, a_{3}, a_{4}$ we have, from (19),

$$
f_{(z)}^{\prime}(x) \equiv 2(1-z) x+\left(a_{1}+a_{2}-z a_{3}-z a_{4}\right) \equiv 0(\bmod p),
$$

from which $a_{1}+a_{2}$ is determined modulo $p$. Then also from (19) we have $f_{(z)}(x) \equiv(1-z) x^{2}+\left(a_{1}+a_{2}-z a_{3}-z a_{4}\right) x+\left(a_{1} a_{2}-z a_{3} a_{4}\right) \equiv 0(\bmod p)$, and so $a_{1} a_{2}$ is also determined modulo $p$. Thus there are at most two choices for $a_{1}, a_{2}$. Use these congruences to eliminate $a_{1}, a_{2}$. We have on writing

$$
\pi_{1}=a_{1}+a_{2}, \quad \pi_{2}=a_{1} a_{2}, \quad \varrho_{1}=a_{3}+a_{4}, \quad \varrho_{2}=a_{3} a_{4},
$$

the identity

$$
\left(\pi_{1}^{2}-4 \pi_{2}\right)\left(B_{1}-B_{2}\right)^{2}-\left(2 B_{2} a_{1}+2 B_{1} a_{2}-\pi_{1}\left(B_{1}+B_{2}\right)\right)^{2}=0 .
$$


Now from (19) we have

$$
\begin{aligned}
\sum_{k=1}^{4} B_{k} \frac{\partial f_{(z)}(x)}{\partial m_{k}} \equiv & \left(B_{1}+B_{2}-z B_{3}-z B_{4}\right) x \\
& +\left(a_{1} B_{2}+a_{2} B_{1}-z a_{3} B_{4}-z a_{4} B_{3}\right) \\
\equiv & 0(\bmod p) .
\end{aligned}
$$

Thus eliminating $a_{1}, a_{2}$ we have

$$
\begin{gathered}
\left(z^{2} \varrho_{1}^{2}-4 z \varrho_{1}(1-z) x-4 x^{2} z(1-z)-4 z \varrho_{2}\right)\left(B_{1}-B_{2}\right)^{2} \\
-z^{2}\left(2\left(B_{3} a_{4}+B_{4} a_{3}\right)+2 x\left(B_{3}+B_{4}\right)-\left(\varrho_{1}+2 x\right)\left(B_{1}+B_{2}\right)\right)^{2} \equiv 0(\bmod p) .
\end{gathered}
$$

Thus from (19),

$$
\begin{aligned}
& (21) \quad S_{6} \ll p^{3} \sum_{\boldsymbol{B}} \#\left\{x, z, a_{3}, a_{4}:\right. \\
& \left(z^{2} \varrho_{1}^{2}-4 z \varrho_{1}(1-z) x-4 x^{2} z(1-z)-4 z \varrho_{2}\right)\left(B_{1}-B_{2}\right)^{2} \\
& \left.-z^{2}\left(2\left(B_{3} a_{4}+B_{4} a_{3}\right)+2 x\left(B_{3}+B_{4}\right)-\left(\varrho_{1}+2 x\right)\left(B_{1}+B_{2}\right)\right)^{2} \equiv 0(\bmod p)\right\} .
\end{aligned}
$$

By Lemma 2, for a given choice of $\boldsymbol{B},(20)$ has at most $O\left(p^{2} \mu\right)$ solutions in $x, z, a_{3}, a_{4}$, unless this polynomial is identically zero modulo $p$. If the coefficient of $z a_{3} a_{4}$ is zero we have

$$
-4\left(B_{1}-B_{2}\right)^{2} \equiv 0(\bmod p),
$$

and thus $B_{1} \equiv B_{2}(\bmod p)$. Under this condition if the coefficient of $z^{2} a_{3}^{2}$ is zero we have

$$
-\left(2 B_{4}-B_{1}-B_{2}\right)^{2} \equiv 0(\bmod p),
$$

and if the coefficient of $z^{2} a_{4}^{2}$ is zero we have

$$
-\left(2 B_{3}-B_{1}-B_{2}\right)^{2} \equiv 0(\bmod p) .
$$

Thus if the polynomial is identically zero modulo $p$ we have

$$
B_{1} \equiv B_{2} \equiv B_{3} \equiv B_{4}(\bmod p) .
$$

Hence the number of such cases is $O\left(\lambda(1+\lambda / p)^{3}\right)$.

Consequently, from (21) we have

$$
S_{6} \ll p^{3}\left(\lambda^{4} p^{2} \mu+\left(\lambda+\frac{\lambda^{4}}{p^{3}}\right) p^{2} \mu^{2}\right) \ll p^{2} h^{4}+p^{5} h^{2} .
$$

The theorem follows from (12), (13) and (16).

5. Introduction to proof of Theorem 2. We may suppose that $p>2$. We consider here the case $r=3$. Noting (11) we write 


$$
\begin{array}{r}
S_{7}=\sum_{m} \#\left\{x, z: 0<x \leq p^{3}, 0<z<p, f_{1}(x) f_{2}(x) \not \equiv 0(\bmod p),\right. \\
f_{(z)}(x) \equiv 0\left(\bmod p^{3}\right), f_{(z)}^{\prime}(x) \equiv 0(\bmod p), \\
\text { either } \left.f_{(z)}^{\prime} \not \equiv 0\left(\bmod p^{2}\right) \text { or } f_{(z)}^{\prime \prime}(x) \not \equiv 0(\bmod p)\right\}
\end{array}
$$

and

$$
\begin{aligned}
& S_{8}=\sum_{\boldsymbol{m}} \#\left\{x, z: 0<x \leq p^{3}, 0<z<p, f_{1}(x) f_{2}(x) \not \equiv 0(\bmod p),\right. \\
& \left.f_{(z)}(x) \equiv 0\left(\bmod p^{3}\right), f_{(z)}^{\prime}(x) \equiv 0\left(\bmod p^{2}\right), f_{(z)}^{\prime \prime}(x) \equiv 0(\bmod p)\right\}
\end{aligned}
$$

so that

$$
S_{4}=p^{2} S_{7}+p^{2} S_{8}
$$

We estimate $S_{7}$ and $S_{8}$ in Section 7 .

We shall use also the polynomials $g_{i}(x)$ given by

$\forall i \leq 3 \quad g_{i}(x)=f_{1}(x) /\left(x+m_{i}\right), \quad \forall i \geq 4 \quad g_{i}(x)=f_{2}(x) /\left(x+m_{i}\right)$.

Thus we have, from (8),

and

$$
f_{(z)}(x)=g_{1}(x)\left(x+m_{1}\right)-z^{p^{2}} g_{4}(x)\left(x+m_{4}\right)
$$

$$
f_{(z)}^{\prime}(x)=g_{1}(x)+g_{2}(x)+g_{3}(x)-z^{p^{2}} g_{4}(x)-z^{p^{2}} g_{5}(x)-z^{p^{2}} g_{6}(x) .
$$

Write

$$
\begin{aligned}
C_{1}(\boldsymbol{m})= & g_{1}(x)\left(x+m_{1}\right)-z g_{4}(x)\left(x+m_{4}\right), \\
C_{2}(\boldsymbol{m})= & \left(2 x+m_{2}+m_{3}\right)\left(x+m_{1}\right)-z\left(2 x+m_{5}+m_{6}\right)\left(x+m_{4}\right) \\
& +\left(g_{1}(x)-z g_{4}(x)\right), \\
C_{3}(\boldsymbol{m})= & 2\left(x+m_{1}\right)-2 z\left(x+m_{4}\right) \\
& +\left(\left(4 x+2 m_{2}+2 m_{3}\right)-z\left(4 x+2 m_{5}+2 m_{6}\right)\right), \\
C_{4}(\boldsymbol{m})= & b_{1} g_{1}(x)+b_{2} g_{2}(x)+b_{3} g_{3}(x)-b_{4} z g_{4}(x)-b_{5} z g_{5}(x)-b_{6} z g_{6}(x) \\
= & \left(b_{2}\left(x+m_{3}\right)+b_{3}\left(x+m_{2}\right)\right)\left(x+m_{1}\right) \\
& -z\left(b_{5}\left(x+m_{6}\right)+b_{6}\left(x+m_{5}\right)\right)\left(x+m_{4}\right)+\left(b_{1} g_{1}(x)-b_{4} z g_{4}(x)\right)
\end{aligned}
$$

and

$$
\begin{aligned}
C_{5}(\boldsymbol{m})= & b_{1}\left(2 x+m_{2}+m_{3}\right)+b_{2}\left(2 x+m_{1}+m_{3}\right)+b_{3}\left(2 x+m_{1}+m_{2}\right) \\
& -b_{4} z\left(2 x+m_{5}+m_{6}\right)-b_{5} z\left(2 x+m_{4}+m_{6}\right) \\
& -b_{6} z\left(2 x+m_{4}+m_{5}\right) \\
= & \left(b_{2}+b_{3}\right)\left(x+m_{1}\right)-z\left(b_{5}+b_{6}\right)\left(x+m_{4}\right) \\
& +\left(b_{1}\left(2 x+m_{2}+m_{3}\right)+b_{2}\left(x+m_{3}\right)+b_{3}\left(x+m_{2}\right)\right. \\
& \left.-z b_{4}\left(2 x+m_{5}+m_{6}\right)-z b_{5}\left(x+m_{6}\right)-z b_{6}\left(x+m_{5}\right)\right) .
\end{aligned}
$$

We define $\lambda$ and $\mu$ by (18). 


\section{Minor lemmas}

LEMma 4. Write

$$
D_{1}=\left|\begin{array}{cc}
g_{1}(x) & -z g_{4}(x) \\
2 x+m_{2}+m_{3} & -z\left(2 x+m_{5}+m_{6}\right)
\end{array}\right| .
$$

Then

$$
\begin{aligned}
\sum_{\substack{\boldsymbol{b} \\
\forall i\left|b_{i}\right|<\lambda}} \#\left\{\boldsymbol{m}, x, z: 0<m_{i} \leq \mu, 0<x \leq p, 0<z<p,\right. \\
\left.f_{1}(x) f_{2}(x) \not \equiv 0(\bmod p), C_{1}(\boldsymbol{m}) \equiv C_{2}(\boldsymbol{m}) \equiv 0(\bmod p), D_{1} \equiv 0(\bmod p)\right\} \\
\ll p \mu^{4} \lambda^{6} .
\end{aligned}
$$

Proof. From $C_{1}(\boldsymbol{m}) \equiv C_{2}(\boldsymbol{m}) \equiv D_{1} \equiv 0(\bmod p)$ it follows that

$$
g_{1}(x) \equiv z g_{4}(x)(\bmod p),
$$

from which $z$ is uniquely determined. Then from $C_{1}(\boldsymbol{m}) \equiv 0(\bmod p)$ it follows that $m_{1}=m_{4}$. Finally,

$$
\begin{aligned}
D_{1}= & z\left(\left(m_{5}+m_{6}-m_{2}-m_{3}\right) x^{2}+2\left(m_{5} m_{6}-m_{2} m_{3}\right) x\right. \\
& \left.+m_{5} m_{6}\left(m_{2}+m_{3}\right)-m_{2} m_{3}\left(m_{5}+m_{6}\right)\right),
\end{aligned}
$$

so that, by Lemma $2, D_{1} / z \equiv 0(\bmod p)$ has $O\left(p \mu^{3}\right)$ solutions as a function of $m_{2}, m_{3}, m_{5}, m_{6}, x$. Thus the required estimate follows trivially.

\section{LEMMA 5. Write}

Then

$$
D_{2}=\left|\begin{array}{ccc}
m_{2} m_{3} & -z m_{5} m_{6} & 0 \\
m_{2}+m_{3} & -z\left(m_{5}+m_{6}\right) & m_{2} m_{3}-z m_{5} m_{6} \\
b_{2} m_{3}+b_{3} m_{2} & -z b_{5} m_{6}-z b_{6} m_{5} & b_{1} m_{2} m_{3}-b_{4} z m_{5} m_{6}
\end{array}\right| .
$$

Pr o of. We have

$$
\begin{aligned}
\sum_{\substack{\boldsymbol{b} \\
\forall i\left|b_{i}\right|<\lambda}} & \\
D_{2} \text { identically } 0(\bmod p) & \left.0<m_{2}, m_{3}, m_{5}, m_{6}, x, z: \mu, 0<x \leq p, 0<z<p\right\} \\
& \ll p^{2} \mu^{4} \lambda\left(\frac{\lambda}{p}+1\right)^{5} .
\end{aligned}
$$

$$
\begin{aligned}
D_{2}= & \left(b_{6}-b_{1}\right) z m_{2}^{2} m_{3}^{2} m_{5}+\left(b_{5}-b_{1}\right) z m_{2}^{2} m_{3}^{2} m_{6}+\left(b_{1}-b_{3}\right) z m_{2}^{2} m_{3} m_{5} m_{6} \\
& +\left(b_{1}-b_{2}\right) z m_{2} m_{3}^{2} m_{5} m_{6}+\left(b_{4}-b_{6}\right) z^{2} m_{2} m_{3} m_{5}^{2} m_{6} \\
& +\left(b_{4}-b_{5}\right) z^{2} m_{2} m_{3} m_{5} m_{6}^{2}+\left(b_{3}-b_{4}\right) z^{2} m_{2} m_{5}^{2} m_{6}^{2} \\
& +\left(b_{2}-b_{4}\right) z^{2} m_{3} m_{5}^{2} m_{6}^{2} .
\end{aligned}
$$

This is identically $0(\bmod p)$ only if

$$
b_{1} \equiv b_{2} \equiv b_{3} \equiv b_{4} \equiv b_{5} \equiv b_{6}(\bmod p) .
$$

The required estimate follows trivially. 
LEMMA 6. We have

$$
\begin{array}{r}
\sum_{\substack{\boldsymbol{b} \\
\forall i\left|b_{i}\right|<\lambda}} \#\left\{\boldsymbol{m}, x, z: 0<m_{i} \leq \mu, 0<x \leq p\right. \\
0<z<p, f_{1}(x) f_{2}(x) \not \equiv 0(\bmod p), \\
\left.C_{1}(\boldsymbol{m}) \equiv C_{2}(\boldsymbol{m}) \equiv C_{3}(\boldsymbol{m}) \equiv 0(\bmod p), D_{1} \equiv 0(\bmod p)\right\} \\
\ll p \mu^{3} \lambda^{6}
\end{array}
$$

where $D_{1}$ is defined by $(25)$.

Proof. From $C_{1}(\boldsymbol{m}) \equiv C_{2}(\boldsymbol{m}) \equiv D_{1} \equiv 0(\bmod p)$ it follows that

$$
g_{1}(x) \equiv z g_{4}(x)(\bmod p),
$$

from which $z$ is uniquely determined. Then from $C_{1}(\boldsymbol{m}) \equiv 0(\bmod p)$ it follows that $m_{1}=m_{4}$ and, since $f_{1}(x) f_{2}(x) \not \equiv 0(\bmod p)$, from $C_{2}(\boldsymbol{m}) \equiv 0$ $(\bmod p)$ that

$$
\left(2 x+m_{2}+m_{3}\right)-z\left(2 x+m_{5}+m_{6}\right) \equiv 0(\bmod p) .
$$

Now substituting into $C_{3}(\boldsymbol{m}) \equiv 0(\bmod p)$ we obtain $z=1$ and so also $m_{2}+m_{3} \equiv m_{5}+m_{6}(\bmod p)$. But also we have $g_{1}(x) \equiv g_{4}(x)(\bmod p)$ and so $m_{2} m_{3} \equiv m_{5} m_{6}(\bmod p)$. Thus $m_{2}, m_{3}$ is a permutation of $m_{5}, m_{6}$. The required estimate follows trivially.

Lemma 7. Write

$$
D_{3}=\left|\begin{array}{ccc}
g_{1}(x) & g_{4}(x) & 0 \\
2 x+m_{2}+m_{3} & 2 x+m_{5}+m_{6} & g_{1}(x) \\
1 & 1 & 2 x+m_{2}+m_{3}
\end{array}\right|
$$

and

$$
D_{4}=\left|\begin{array}{ccc}
g_{1}(x) & g_{4}(x) & 0 \\
2 x+m_{2}+m_{3} & 2 x+m_{5}+m_{6} & g_{4}(x) \\
1 & 1 & 2 x+m_{5}+m_{6}
\end{array}\right|
$$

Then

$$
\begin{aligned}
\sum_{\substack{\boldsymbol{b} \\
\forall i\left|b_{i}\right|<\lambda}} \#\left\{m_{2}, m_{3}, m_{5}, m_{6}, x:\right. & \\
0<m_{i} \leq \mu, 0<x \leq p, D_{3} \equiv D_{4} \equiv 0 & (\bmod p)\} \\
& \ll p \mu^{2} \lambda^{6} .
\end{aligned}
$$

Proof. The conditions $D_{3} \equiv D_{4} \equiv 0(\bmod p)$ expand to give

$$
\begin{aligned}
& \left(g_{1}(x)-g_{4}(x)\right)\left(\left(2 x+m_{5}+m_{6}\right)\left(2 x+m_{2}+m_{3}\right)-g_{1}(x)\right) \\
& \quad-g_{4}(x)\left(m_{2}+m_{3}-m_{5}-m_{6}\right)\left(2 x+m_{2}+m_{3}\right) \\
& \equiv\left(g_{1}(x)-g_{4}(x)\right)\left(\left(2 x+m_{5}+m_{6}\right)^{2}-g_{4}(x)\right) \\
& \quad-g_{4}(x)\left(m_{2}+m_{3}-m_{5}-m_{6}\right)\left(2 x+m_{5}+m_{6}\right) \equiv 0(\bmod p) .
\end{aligned}
$$


These will have only $O(1)$ solutions $x$ unless both polynomials are identically $0(\bmod p)$. But in the first of these the coefficient of $x^{3}$ is $m_{2}+m_{3}-m_{5}-m_{6}$, and if this is $0(\bmod p)$ then the coefficient of $x^{2}$ is $3\left(m_{2} m_{3}-m_{5} m_{6}\right)$. Thus if both polynomials in $x$ are identically $0(\bmod p)$ then the pair $m_{2}, m_{3}$ is a permutation of $m_{5}, m_{6}$. This contributes

$$
\ll \lambda^{6} p \mu^{2}
$$

to our estimate.

Now consider the other case in which at least one of $D_{3}$ and $D_{4}$ is not identically $0(\bmod p)$. Then there are only $O(1)$ values for $x$. The two polynomial congruences $D_{3} \equiv D_{4} \equiv 0(\bmod p)$ are cubics. The difference between these polynomials is

$$
\left|\begin{array}{ccc}
g_{1}(x) & g_{4}(x) & 0 \\
2 x+m_{2}+m_{3} & 2 x+m_{5}+m_{6} & g_{1}(x)-g_{4}(x) \\
1 & 1 & m_{2}+m_{3}-m_{5}-m_{6}
\end{array}\right| .
$$

By row and column operations this simplifies to

$$
\left|\begin{array}{ccc}
m_{2} m_{3} & m_{5} m_{6} & 0 \\
m_{2}+m_{3} & m_{5}+m_{6} & m_{2} m_{3}-m_{5} m_{6} \\
1 & 1 & m_{2}+m_{3}-m_{5}-m_{6}
\end{array}\right|
$$

which is a polynomial in $m_{2}, m_{3}, m_{5}, m_{6}$. This polynomial is -1 when $m_{2}=$ $m_{3}=1, m_{5}=m_{6}=0$. Thus it is not identically $0(\bmod p)$ and so has $O\left(\mu^{3}\right)$ solutions in $m_{2}, m_{3}, m_{5}, m_{6}$. Thus this contributes

$$
\ll \lambda^{6} \mu^{3}
$$

to our estimate. The lemma follows from (26) and (27).

Lemma 8. Write

$$
\begin{aligned}
& D_{5}=\left|\begin{array}{ccc}
m_{2} m_{3} & -m_{5} m_{6} & 0 \\
m_{2}+m_{3} & -\left(m_{5}+m_{6}\right) & m_{2} m_{3} \\
b_{2} m_{3}+b_{3} m_{2} & -b_{5} m_{6}-b_{6} m_{5} & b_{1} m_{2} m_{3}
\end{array}\right| \\
& \times\left|\begin{array}{ccc}
m_{2} m_{3}-m_{5} m_{6} & m_{5} m_{6} & 0 \\
m_{2}+m_{3}-m_{5}-m_{6} & m_{5}+m_{6} & m_{5} m_{6} \\
0 & 1 & m_{5}+m_{6}
\end{array}\right| \\
& -\left|\begin{array}{ccc}
m_{2} m_{3} & -m_{5} m_{6} & 0 \\
m_{2}+m_{3} & -\left(m_{5}+m_{6}\right) & m_{5} m_{6} \\
b_{2} m_{3}+b_{3} m_{2} & -b_{5} m_{6}-b_{6} m_{5} & b_{4} m_{5} m_{6}
\end{array}\right| \\
& \times\left|\begin{array}{ccc}
m_{2} m_{3}-m_{5} m_{6} & m_{5} m_{6} & 0 \\
m_{2}+m_{3}-m_{5}-m_{6} & m_{5}+m_{6} & m_{2} m_{3} \\
0 & 1 & m_{2}+m_{3}
\end{array}\right| \text {. }
\end{aligned}
$$


Then

$$
\sum_{\substack{\forall i\left|b_{i}\right|<\lambda \\ D_{5} \text { identically } 0(\bmod p)}} \#\left\{m_{2}, m_{3}, m_{5}, m_{6}: 0<m_{i} \leq \mu\right\} \ll \mu^{4} \lambda\left(\frac{\lambda}{p}+1\right)^{5} .
$$

Proof. Substitute $m_{6}=0$ in $D_{5}$ to obtain $\left(b_{6}-b_{1}\right) m_{2}^{3} m_{3}^{3} m_{5}^{3}$. Thus if $D_{5}$ is identically $0(\bmod p)$ then $b_{6} \equiv b_{1}(\bmod p)$. Similar arguments give

$$
b_{1} \equiv b_{5} \equiv b_{6}, \quad b_{2} \equiv b_{3} \equiv b_{4}(\bmod p) .
$$

Substituting this in $D_{5}$, and putting $m_{2}=m_{3}=m_{5}=1$ we obtain

$$
-\left(b_{4}-b_{1}\right)\left(1-m_{6}\right)^{2} m_{6}
$$

also. The required estimate follows trivially.

\section{Proof of Theorem 2}

Lemma 9. We have

$$
S_{7} \ll h^{6}+p^{3} h \mu^{3}
$$

where $\mu$ is defined by (18).

Proof. From Lemma 1 applied to (22) it follows that

$$
\begin{aligned}
S_{7} \ll p \sum_{\boldsymbol{m}} \#\left\{z: 0<z<p, \exists x f_{1}(x) f_{2}(x) \not \equiv 0(\bmod p),\right. \\
\left.f_{(z)}(x) \equiv 0\left(\bmod p^{2}\right), f_{(z)}^{\prime}(x) \equiv 0(\bmod p)\right\} .
\end{aligned}
$$

We can rewrite this as

$$
\begin{aligned}
S_{7} \ll p \sum_{x=1}^{p} \sum_{z=1}^{p-1} \#\left\{\boldsymbol{m}: f_{1}(x) f_{2}(x) \not \equiv 0(\bmod p),\right. \\
\left.f_{(z)}(x) \equiv 0\left(\bmod p^{2}\right), f_{(z)}^{\prime}(x) \equiv 0(\bmod p)\right\},
\end{aligned}
$$

say. Write

$$
\begin{array}{r}
N=\#\left\{\boldsymbol{m}: f_{1}(x) f_{2}(x) \not \equiv 0(\bmod p), f_{(z)}(x) \equiv 0\left(\bmod p^{2}\right),\right. \\
\left.f_{(z)}^{\prime}(x) \equiv 0(\bmod p)\right\} .
\end{array}
$$

Thus by Lemma 3,

$$
\begin{aligned}
N \ll \sum_{\substack{\boldsymbol{b} \\
\forall i\left|b_{i}\right|<\lambda}} \#\left\{\boldsymbol{m}: \forall i 0<m_{i} \leq \mu, f_{1}(x) f_{2}(x) \not \equiv 0(\bmod p),\right. \\
\left.0 \equiv C_{1}(\boldsymbol{m}) \equiv C_{2}(\boldsymbol{m}) \equiv C_{4}(\boldsymbol{m})(\bmod p)\right\}
\end{aligned}
$$

if $\lambda>1$, and follows immediately from (29) if $\lambda=1$. Thus from (28) we have

$$
\begin{aligned}
S_{7} \ll p & \sum_{\substack{\boldsymbol{b} \\
\forall i\left|b_{i}\right|<\lambda}} \#\left\{\boldsymbol{m}, x, z: 0<m_{i} \leq \mu, 0<x \leq p, 0<z<p\right. \\
& \left.f_{1}(x) f_{2}(x) \not \equiv 0(\bmod p), 0 \equiv C_{1}(\boldsymbol{m}) \equiv C_{2}(\boldsymbol{m}) \equiv C_{4}(\boldsymbol{m})(\bmod p)\right\} .
\end{aligned}
$$


But by Lemma 4,

Thus

$$
\begin{aligned}
& \sum_{\substack{\boldsymbol{b} \\
\forall i\left|b_{i}\right|<\lambda}} \#\left\{\boldsymbol{m}, x, z: 0<m_{i} \leq \mu, 0<x \leq p, 0<z<p,\right. \\
& f_{1}(x) f_{2}(x) \not \equiv 0(\bmod p), 0 \equiv C_{1}(\boldsymbol{m}) \equiv\left.C_{2}(\boldsymbol{m}) \equiv D_{1}(\bmod p)\right\} \\
& \ll p \mu^{4} \lambda^{6} \ll \frac{h^{6}}{p}+p h^{4} .
\end{aligned}
$$

where

$$
S_{7} \ll\left(p \sum_{\substack{\boldsymbol{b} \\ \forall i\left|b_{i}\right|<\lambda}} \# W_{1}\right)+h^{6}+p^{2} h^{4}
$$

$W_{1}=\left\{\boldsymbol{m}, x, z: 0<m_{i} \leq \mu, 0<x \leq p, 0<z<p\right.$,

$$
\left.f_{1}(x) f_{2}(x) \not \equiv 0(\bmod p), 0 \equiv C_{1}(\boldsymbol{m}) \equiv C_{2}(\boldsymbol{m}) \equiv C_{4}(\boldsymbol{m}) \not \equiv D_{1}(\bmod p)\right\} .
$$

Consider $(\boldsymbol{m}, x, z) \in W_{1}$. Given $m_{2}, m_{3}, m_{5}, m_{6}, z, x$, the values of $m_{1}$, $m_{4}$ are uniquely determined by $C_{1}(\boldsymbol{m}) \equiv C_{2}(\boldsymbol{m}) \equiv 0(\bmod p)$ since $D_{1} \not \equiv 0$ $(\bmod p)$. Eliminating $m_{1}, m_{4}$ from $C_{1}(\boldsymbol{m}) \equiv C_{2}(\boldsymbol{m}) \equiv C_{4}(\boldsymbol{m}) \equiv 0(\bmod p)$ we obtain

$$
\begin{aligned}
D(x) & =\left|\begin{array}{ccc}
g_{1}(x) & -z g_{4}(x) & 0 \\
2 x+m_{2}+m_{3} & -z\left(2 x+m_{5}+m_{6}\right) & g_{1}(x)-z g_{4}(x) \\
b_{2}\left(x+m_{3}\right)+b_{3}\left(x+m_{2}\right) & -z b_{5}\left(x+m_{6}\right)-z b_{6}\left(x+m_{5}\right) & b_{1} g_{1}(x)-b_{4} z g_{4}(x)
\end{array}\right| \\
& \equiv 0(\bmod p) .
\end{aligned}
$$

By Lemma 2 this has $O\left(p^{2} \mu^{3}\right)$ solutions in $m_{2}, m_{3}, m_{5}, m_{6}, z, x$ unless it is identically $0(\bmod p)$ as a polynomial in these variables. In the latter case $D(0)$ will also be identically $0(\bmod p)$. However, we have

$$
D(0)=\left|\begin{array}{ccc}
m_{2} m_{3} & -z m_{5} m_{6} & 0 \\
m_{2}+m_{3} & -z\left(m_{5}+m_{6}\right) & m_{2} m_{3}-z m_{5} m_{6} \\
b_{2} m_{3}+b_{3} m_{2} & -z b_{5} m_{6}-z b_{6} m_{5} & b_{1} m_{2} m_{3}-b_{4} z m_{5} m_{6}
\end{array}\right|,
$$

and by Lemma 5 ,

$$
\begin{array}{r}
\sum_{\substack{\boldsymbol{b} \\
\forall i\left|b_{i}\right|<\lambda}} \#\left\{m_{2}, m_{3}, m_{5}, m_{6}, x, z:\right. \\
\left.0<m_{i} \leq \mu, 0<x \leq p, 0<z<p\right\} \\
\quad \ll p^{2} \mu^{4} \lambda\left(\frac{\lambda}{p}+1\right)^{5} .
\end{array}
$$

Thus, by (31),

$$
S_{7} \ll p\left(\lambda^{6} p^{2} \mu^{3}+\lambda\left(\frac{\lambda}{p}+1\right)^{5} p^{2} \mu^{4}\right)+h^{6}+p^{2} h^{4} \ll h^{6}+p^{3} h \mu^{3},
$$

which completes the proof of the lemma. 
Lemma 10. We have

$$
S_{8} \ll h^{6}+p^{3} h \mu^{3}+p^{4} \mu^{2} .
$$

Pr o of. We have, from (23),

$$
\begin{aligned}
S_{8}=p^{2} \sum_{\boldsymbol{m}} \#\left\{x, z: 0<x \leq p, 0<z<p, f_{1}(x) f_{2}(x) \not \equiv 0(\bmod p),\right. \\
\left.f_{(z)}(x) \equiv 0\left(\bmod p^{3}\right), f_{(z)}^{\prime}(x) \equiv 0\left(\bmod p^{2}\right), f_{(z)}^{\prime \prime}(x) \equiv 0(\bmod p)\right\} .
\end{aligned}
$$

Rewrite this as

$$
\begin{aligned}
S_{8} & \ll p^{2} \sum_{x=1}^{p} \sum_{z=1}^{p-1} \#\left\{\boldsymbol{m}: f_{1}(x) f_{2}(x) \not \equiv 0(\bmod p),\right. \\
f_{(z)}(x) & \left.\equiv 0\left(\bmod p^{3}\right), f_{(z)}^{\prime}(x) \equiv 0\left(\bmod p^{2}\right), f_{(z)}^{\prime \prime}(x) \equiv 0(\bmod p)\right\} .
\end{aligned}
$$

Write

$N=\#\left\{\boldsymbol{m}: f_{1}(x) f_{2}(x) \not \equiv 0(\bmod p), f_{(z)}(x) \equiv 0\left(\bmod p^{2}\right)\right.$,

$$
\left.f_{(z)}^{\prime}(x) \equiv 0\left(\bmod p^{2}\right), f_{(z)}^{\prime \prime}(x) \equiv 0(\bmod p)\right\} .
$$

Define $\lambda$ and $\mu$ by (18). Thus by Lemma 3 we have

$$
\begin{aligned}
& N \ll \sum_{\substack{\boldsymbol{b} \\
\forall i\left|b_{i}\right|<\lambda}} \#\left\{\boldsymbol{m}: \forall i 0<m_{i} \leq \mu, f_{1}(x) f_{2}(x) \not \equiv 0(\bmod p),\right. \\
& \left.0 \equiv C_{1}(\boldsymbol{m}) \equiv C_{2}(\boldsymbol{m}) \equiv C_{3}(\boldsymbol{m}) \equiv C_{4}(\boldsymbol{m}) \equiv C_{5}(\boldsymbol{m})(\bmod p)\right\} .
\end{aligned}
$$

By Lemma 6,

$$
\begin{aligned}
& \sum_{\substack{\boldsymbol{b} \\
\forall i\left|b_{i}\right|<\lambda}} \#\left\{\boldsymbol{m}, x, z: 0<m_{i} \leq \mu, 0<x \leq p, 0<z<p\right. \\
&\left.f_{1}(x) f_{2}(x) \not \equiv 0(\bmod p), C_{1}(\boldsymbol{m}) \equiv C_{2}(\boldsymbol{m}) \equiv C_{3}(\boldsymbol{m}) \equiv D_{1} \equiv 0(\bmod p)\right\} \\
& \ll p \mu^{3} \lambda^{6} \ll \frac{h^{6}}{p^{2}}+p h^{3} .
\end{aligned}
$$

Thus by (32) we have

$$
S_{8} \ll\left(p^{2} \sum_{\substack{\boldsymbol{b} \\ \forall i\left|b_{i}\right|<\lambda}} \# W_{2}\right)+h^{6}+p^{3} \mu^{3},
$$

where

$$
\begin{aligned}
& W_{2}=\left\{\boldsymbol{m}, x, z: 0<m_{i} \leq \mu, 0<x \leq p, 0<z<p\right. \\
& f_{1}(x) f_{2}(x) \not \equiv 0(\bmod p), \\
&\left.0 \equiv C_{1}(\boldsymbol{m}) \equiv C_{2}(\boldsymbol{m}) \equiv C_{3}(\boldsymbol{m}) \equiv C_{4}(\boldsymbol{m}) \equiv C_{5}(\boldsymbol{m}) \not \equiv D_{1}(\bmod p)\right\}
\end{aligned}
$$

Consider $(\boldsymbol{m}, x, z) \in W_{2}$. Given $m_{2}, m_{3}, m_{5}, m_{6}, z, x$, the values of $m_{1}$, $m_{4}$ are uniquely determined by $C_{1}(\boldsymbol{m}) \equiv C_{2}(\boldsymbol{m}) \equiv 0(\bmod p)$ since $D_{1} \not \equiv 0$ 
$(\bmod p)$. Eliminating $m_{1}, m_{4}$ from $C_{1}(\boldsymbol{m}) \equiv C_{2}(\boldsymbol{m}) \equiv C_{4}(\boldsymbol{m}) \equiv 0(\bmod p)$ we obtain

$$
\begin{aligned}
E_{1} & =\left|\begin{array}{ccc}
g_{1}(x) & -g_{4}(x) & 0 \\
2 x+m_{2}+m_{3} & -\left(2 x+m_{5}+m_{6}\right) & g_{1}(x)-z g_{4}(x) \\
b_{2}\left(x+m_{3}\right)+b_{3}\left(x+m_{2}\right) & -b_{5}\left(x+m_{6}\right)-b_{6}\left(x+m_{5}\right) & b_{1} g_{1}(x)-b_{4} z g_{4}(x)
\end{array}\right| \\
& \equiv 0(\bmod p) .
\end{aligned}
$$

Also eliminating $m_{1}, m_{4}$ from $C_{1}(\boldsymbol{m}) \equiv C_{2}(\boldsymbol{m}) \equiv C_{3}(\boldsymbol{m}) \equiv 0(\bmod p)$ we obtain

$$
\begin{aligned}
E_{2} & =\left|\begin{array}{ccc}
g_{1}(x) & -g_{4}(x) & 0 \\
2 x+m_{2}+m_{3} & -\left(2 x+m_{5}+m_{6}\right) & g_{1}(x)-z g_{4}(x) \\
2 & -2 & 2\left(\left(2 x+m_{2}+m_{3}\right)-z\left(2 x+m_{5}+m_{6}\right)\right)
\end{array}\right| \\
& \equiv 0(\bmod p),
\end{aligned}
$$

which can be rewritten as

$$
D_{3} \equiv z D_{4}(\bmod p)
$$

But by Lemma 7 ,

$$
\begin{aligned}
& \sum_{\substack{\boldsymbol{b} \\
\forall i\left|b_{i}\right|<\lambda}} \#\left\{m_{2}, m_{3}, m_{5}, m_{6}, x: 0<m_{i} \leq \mu, 0<x\right. \leq p, \\
&\left.D_{3} \equiv D_{4} \equiv 0(\bmod p)\right\} \\
& \ll p \mu^{2} \lambda^{6} \ll \frac{h^{6}}{p^{3}}+p \mu^{2} .
\end{aligned}
$$

Thus, by (33),

$$
S_{8} \ll p^{2}\left(\sum_{\substack{\boldsymbol{b} \\ \forall i\left|b_{i}\right|<\lambda}} \# W_{3}\right)+h^{6}+p^{4} \mu^{2}
$$

where

$W_{3}=\left\{m_{2}, m_{3}, m_{5}, m_{6}, x, z: 0<m_{i} \leq \mu, 0<x \leq p, 0<z<p\right.$,

$$
\left.E_{1} \equiv 0(\bmod p), D_{3} \equiv z D_{4}(\bmod p), D_{3}, D_{4} \text { not both } 0(\bmod p)\right\} .
$$

Now, for $\left(m_{2}, m_{3}, m_{5}, m_{6}, x, z\right) \in W_{3}, z$ is uniquely determined by (36). Also (34) can be rewritten as

$$
D_{6} \equiv z D_{7}(\bmod p)
$$

where

$$
D_{6}=\left|\begin{array}{ccc}
g_{1}(x) & -g_{4}(x) & 0 \\
2 x+m_{2}+m_{3} & -\left(2 x+m_{5}+m_{6}\right) & g_{1}(x) \\
b_{2}\left(x+m_{3}\right)+b_{3}\left(x+m_{2}\right) & -b_{5}\left(x+m_{6}\right)-b_{6}\left(x+m_{5}\right) & b_{1} g_{1}(x)
\end{array}\right|
$$


and

$$
D_{7}=\left|\begin{array}{ccc}
g_{1}(x) & -g_{4}(x) & 0 \\
2 x+m_{2}+m_{3} & -\left(2 x+m_{5}+m_{6}\right) & g_{4}(x) \\
b_{2}\left(x+m_{3}\right)+b_{3}\left(x+m_{2}\right) & -b_{5}\left(x+m_{6}\right)-b_{6}\left(x+m_{5}\right) & b_{4} g_{4}(x)
\end{array}\right| .
$$

Thus, by (37), we have

$$
S_{8} \ll p^{2}\left(\sum_{\substack{\boldsymbol{b} \\ \forall i\left|b_{i}\right|<\lambda}} \# W_{4}\right)+h^{6}+p^{4} \mu^{2},
$$

where

$$
\begin{aligned}
& W_{4}=\left\{m_{2}, m_{3}, m_{5}, m_{6}, x: 0<m_{i} \leq \mu, 0<x \leq p,\right. \\
&\left.D_{3} D_{7} \equiv D_{4} D_{6}(\bmod p)\right\} .
\end{aligned}
$$

Write

$$
H(x)=D_{3} D_{7}-D_{4} D_{6} .
$$

Then $H(0)=D_{5}$ and so $H(x)$ can be identically $0(\bmod p)$ only if $D_{5}$ is. But by Lemma 8 ,

$$
\begin{aligned}
\sum_{\substack{\boldsymbol{b} \\
\forall i\left|b_{i}\right|<\lambda}} \#\left\{m_{2}, m_{3}, m_{5}, m_{6}: 0<m_{i} \leq \mu, H(x) \text { is identically } 0(\bmod p)\right\} \\
\ll \mu^{4} \lambda\left(\frac{\lambda}{p}+1\right)^{5} \ll \frac{h^{6}}{p^{7}}+h \mu^{3} .
\end{aligned}
$$

Thus by (38)

$$
\text { (39) } \quad S_{8} \ll p^{2}\left(\sum_{\substack{\forall i\left|b_{i}\right|<\lambda \\ H(x) \text { not identically } 0(\bmod p)}} \# W_{5}\right)+h^{6}+p^{3} h \mu^{3}+p^{4} \mu^{2},
$$

where

$$
W_{5}=\left\{m_{2}, m_{3}, m_{5}, m_{6}, x: 0<m_{i} \leq \mu, 0<x \leq p, H(x) \equiv 0(\bmod p)\right\} .
$$

By Lemma 2,

$$
\# W_{5} \ll p \mu^{3},
$$

and thus by (39),

$$
S_{8} \ll p^{3} \mu^{3} \lambda^{6}+p^{4} \mu^{2}+h^{6}+p^{3} h \mu^{3} \ll h^{6}+p^{3} h \mu^{3}+p^{4} \mu^{2},
$$

which completes the proof of the lemma.

Proof of Theorem 2. Follows from Lemmas 9 and 10.

8. Corollaries. In [3] it was shown that from (1) it follows that

$$
\left|\sum_{x=N+1}^{N+H} \chi(x)\right| \ll H^{1 / 2} k^{3 / 16+\varepsilon} \text {. }
$$


More generally, in [3] it was shown that if $k$ is cubefree and $r \geq 2$ then

$$
\left|\sum_{x=N+1}^{N+H} \chi(x)\right| \ll H^{1-1 / r} k^{(r+1) /\left(4 r^{2}\right)+\varepsilon} .
$$

It would follow from (3) that

$$
\sum_{\text {primitive } \chi}\left|\sum_{x=N+1}^{N+H} \chi(x)\right| \ll k k^{1 / 4+\varepsilon} H^{1 / 4} .
$$

Similarly from (4) it would follow that

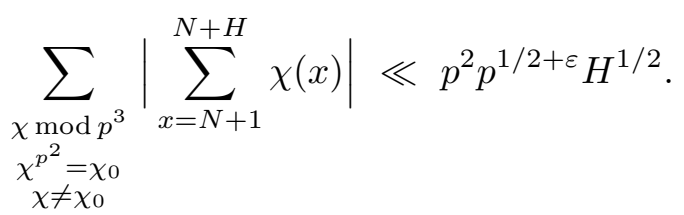

This estimate is improved by the following corollaries.

Corollary 1. If $H \leq p^{3 / 2}$ then

$$
\sum_{\substack{\chi \bmod _{p^{3}} \\ \chi^{p^{2}}=\chi_{0} \\ \chi \neq \chi_{0}}}\left|\sum_{x=N+1}^{N+H} \chi(x)\right| \ll p^{2} p^{3 / 4} H^{1 / 4} .
$$

Proof. From the proof of Lemma 2 of [5] we see that if $2^{\nu}<p^{3}$ then

$$
\begin{aligned}
\left|\sum_{x=N+1}^{N+H} \chi(x)\right| \leq & +H^{3 / 4} 2^{-\nu}\left(\sum_{m=1}^{p^{3}}\left|\sum_{x=m+1}^{x+2^{\nu}} \chi(x)\right|^{4}\right)^{1 / 4} \\
& +\sum_{\mu=0}^{\nu-1} 2^{-\mu / 4}\left(\sum_{m=1}^{p^{3}}\left|\sum_{x=m+1}^{x+2^{\mu}} \chi(x)\right|^{4}\right)^{1 / 4} .
\end{aligned}
$$

Choose $H / 2<2^{\nu}<H$. Then we have

$$
\begin{aligned}
& \sum_{\chi \bmod p^{3}}\left|\sum_{x=N+1}^{N+H} \chi(x)\right| \ll p^{2}+H^{3 / 4} 2^{-\nu} \sum_{\chi}\left(\sum_{m=1}^{p^{3}}\left|\sum_{x=m+1}^{x+2^{\nu}} \chi(x)\right|^{4}\right)^{1 / 4} \\
& \begin{array}{c}
\chi^{p^{2}}=\chi_{0} \\
\chi \neq \chi_{0}
\end{array} \\
& +\sum_{\mu=0}^{\nu-1} 2^{-\mu / 4} \sum_{\chi}\left(\sum_{m=1}^{p^{3}}\left|\sum_{x=m+1}^{x+2^{\mu}} \chi(x)\right|^{4}\right)^{1 / 4}
\end{aligned}
$$




$$
\begin{aligned}
& \ll p^{2}+H^{3 / 4} 2^{-\nu} p^{3 / 2}\left(\sum_{\chi} \sum_{m=1}^{p^{3}}\left|\sum_{x=m+1}^{x+2^{\nu}} \chi(x)\right|^{4}\right)^{1 / 4} \\
& +\sum_{\mu=0}^{\nu-1} 2^{-\mu / 4} p^{3 / 2}\left(\sum_{\chi} \sum_{m=1}^{p^{3}}\left|\sum_{x=m+1}^{x+2^{\mu}} \chi(x)\right|^{4}\right)^{1 / 4} \\
& \ll p^{2}+H^{3 / 4} 2^{-\nu} p^{3 / 2}\left(p^{2} 2^{4 \nu}+p^{5} 2^{2 \nu}\right)^{1 / 4} \\
& +\sum_{\mu=0}^{\nu-1} 2^{-\mu / 4} p^{3 / 2}\left(p^{2} 2^{4 \mu}+p^{5} 2^{2 \mu}\right)^{1 / 4} \\
& \ll H^{1 / 4} p^{11 / 4} .
\end{aligned}
$$

Corollary 2. If $H \geq p^{3 / 2}$ then

$$
\sum_{\substack{\chi \bmod p^{3} \\ \chi^{p^{2}}=\chi_{0} \\ \chi \neq \chi_{0}}}\left|\sum_{x=N+1}^{N+H} \chi(x)\right| \ll p^{2} p^{3 / 8+\delta} H^{1 / 2} .
$$

Proof. From Lemma 6 of [3], with $p^{3 / 2}<2^{\nu} \leq 2 p^{3 / 2}$, it follows that for $p^{3 / 2+\delta} \leq H \leq p^{9 / 4-\delta}$ there is an $h$ satisfying $1 \leq h \leq 2^{\nu}$ for which

$$
\begin{aligned}
& \quad\left|\sum_{x=N+1}^{N+H} \chi(x)\right| \\
& \quad \ll \max \left(H^{1 / 2} p^{-3 / 4} h^{-1 / 4}(\log p)\left(\sum_{x=1}^{p^{3}}\left|\sum_{m=1}^{h} \chi(x+m)\right|^{4}\right)^{1 / 4}, H p^{-3 / 2}\right) .
\end{aligned}
$$

From this it follows that

$$
\begin{aligned}
& \sum_{\substack{\chi \bmod p^{3} \\
\chi^{p^{2}}=\chi_{0} \\
\chi \neq \chi_{0}}}\left|\sum_{x=N+1}^{N+H} \chi(x)\right| \\
& \qquad \sum_{\mu=0}^{\nu} H^{1 / 2} p^{-3 / 4} 2^{-\mu / 4}(\log p) p^{3 / 2}\left(p^{2} 2^{4 \mu}+p^{5} 2^{2 \mu}\right)^{1 / 4}+H p^{1 / 2} \\
& \ll p^{2} p^{3 / 8} H^{1 / 2} \log p .
\end{aligned}
$$

Corollary 3. If $p<H<p^{6 / 5}$ then

$$
\sum_{\substack{\chi \bmod _{p^{3}} \\ \chi^{p^{2}}=\chi_{0} \\ \chi \neq \chi_{0}}}\left|\sum_{x=N+1}^{N+H} \chi(x)\right| \ll p^{2} p^{1+\varepsilon} .
$$


Proof. Choose $H / 2<2^{\nu} \leq H$ and apply Theorem 2 in the proof of Corollary 1.

Corollary 4. If $H \geq p^{6 / 5}$ then

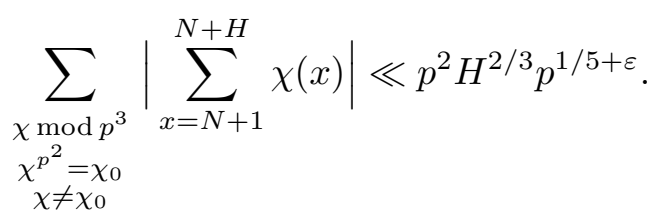

Proof. Choose $p^{6 / 5}<2^{\nu} \leq 2 p^{6 / 5}$ and apply Theorem 2 in the proof of Corollary 2.

\section{References}

[1] D. A. Burgess, On character sums and primitive roots, Proc. London Math. Soc. (3) 12 (1962), 179-192.

[2] -, On character sums and L-series, ibid. 12 (1962), 193-206.

[3] -, On character sums and L-series II, ibid. 13 (1963), 524-536.

[4] -, Estimation of character sums modulo a small power of a prime, J. London Math. Soc. (2) 30 (1984), 385-393.

[5] —, Mean values of character sums, Mathematika 33 (1986), 1-5.

[6] -, Mean values of character sums II, ibid. 34 (1987), 1-7.

[7] -, On a set of congruences related to character sums III, J. London Math. Soc. (2) 45 (1992), 201-214.

[8] —, Mean values of character sums III, Mathematika 42 (1995), 133-136.

Department of Mathematics

The University

Nottingham, NG7 2RD, U.K.

E-mail: dab@maths.nott.ac.uk 